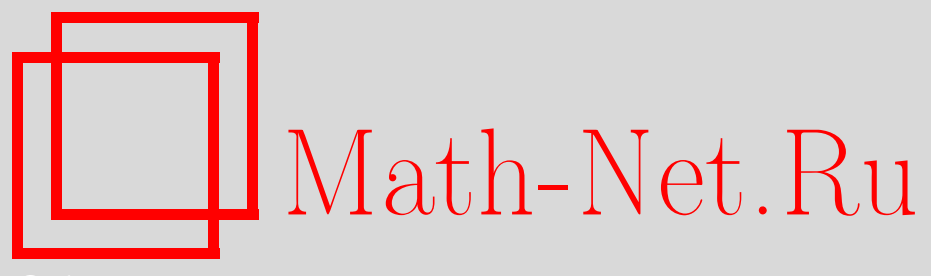

В. Н. Дубинин, Симметризация Штейнера и начальные коэффициенты однолистных функций, Изв. РАН. Сер. матем., 2010, том 74, выпуск 4, 75-82

DOI: https://doi.org/10.4213/im4080

Использование Общероссийского математического портала Math-Net.Ru подразумевает, что вы прочитали и согласны с пользовательским соглашением http://www.mathnet.ru/rus/agreement

Параметры загрузки:

IP : 107.22 .136 .117

26 апреля 2023 г., 10:35:56

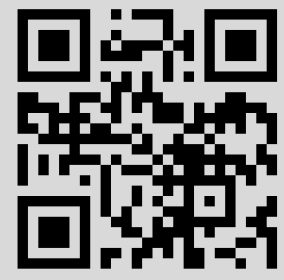


УДК 517.54

\author{
В. Н. Дубинин
}

\title{
Симметризация Штейнера и начальные коэффициенты однолистных функций
}

\begin{abstract}
Для начальных коэффициентов мероморфной и однолистной в области $D=\{z:|z|>1\}$ функции $f(z)=a_{1} z+a_{0}+a_{-1} / z+\cdots$ установлено неравенство $\left|a_{1}\right|^{2}-\operatorname{Re} a_{1} a_{-1} \geqslant\left|a_{1}^{*}\right|^{2}-\operatorname{Re} a_{1}^{*} a_{-1}^{*}$, где $a_{1}^{*}$ и $a_{-1}^{*}-$ соответствующие коэффициенты из разложения функции $f^{*}(z)$, конформно и однолистно отображающей область $D$ на внешность результата симметризации Штейнера относительно вещественной оси дополнения множества $f(D)$. Ранее было известно неравенство Полиа и Сегё $\left|a_{1}\right| \geqslant\left|a_{1}^{*}\right|$. Приводятся некоторые приложения полученного неравенства для функций класса $\Sigma$.

Библиография: 7 наименований.
\end{abstract}

Ключевые слова: симметризация Штейнера, емкость множества, однолистные функции, теоремы покрытия.

\section{§ 1. Введение и формулировки результатов}

Для произвольного вещественного $u$ обозначим через $l(u)$ вертикальную прямую $\operatorname{Re} w=u$. Cuмметризацией Штейнера замкнутого ограниченного множества $E \subset \mathbb{C}_{w}$ относительно вещественной оси называется преобразование этого множества в симметричное множество $E^{*}$, определенное следующим образом:

$$
E^{*}=\{w=u+i v: E \cap l(u) \neq \varnothing, 2|v| \leqslant \mu(E \cap l(u))\},
$$

где $\mu$ - линейная мера Лебега. Пусть $E$ - невырожденный континуум, и пусть функция

$$
f(z)=a_{1} z+a_{0}+\frac{a_{-1}}{z}+\cdots
$$

конформно и однолистно отображает внешность круга $D:=\{z:|z|>1\}$ на связную компоненту дополнения $\overline{\mathbb{C}}_{w} \backslash E$, содержащую бесконечно удаленную точку. Обозначим через

$$
f^{*}(z)=a_{1}^{*} z+a_{0}^{*}+\frac{a_{-1}^{*}}{z}+\cdots
$$

функцию, конформно и однолистно отображающую область $D$ на $\overline{\mathbb{C}}_{w} \backslash E^{*}$. Хорошо известно неравенство Полиа и Сеге [1]

$$
\left|a_{1}\right| \geqslant\left|a_{1}^{*}\right|
$$

Работа выполнена при финансовой поддержке РФФИ (грант № 08-01-00028) и ДВО РАН (грант № 09-III-A-01-007).

(C) В. Н. Дувинин, 2010 
эквивалентное неравенству для логарифмических емкостей

$$
\operatorname{cap} E \geqslant \operatorname{cap} E^{*} \text {. }
$$

Естественно поставить вопрос о поведении последующих коэффициентов в разложении функции $f$ при симметризации Штейнера. Не существует содержательного неравенства между $\left|a_{0}\right|$ и $\left|a_{0}^{*}\right|$. Действительно, сдвиг множества $E$ вдоль мнимой оси не меняет $\operatorname{Re} a_{0}, \operatorname{Re} a_{0}^{*}$ и $\operatorname{Im} a_{0}^{*}=0$ и делает любым значение $\operatorname{Im} a_{0}$. С другой стороны, можно привести примеры, когда $\operatorname{Re} a_{0} \neq \operatorname{Re} a_{0}^{*}$, и в этом случае сдвиг множества $E$ вдоль вещественной оси приводит к неравенству $\left|\operatorname{Re} a_{0}\right|<\left|\operatorname{Re} a_{0}^{*}\right|$. Далее, если множество $E$ есть отрезок, наклоненный к вещественной оси под острым углом, то $\left|a_{-1}\right|>\left|a_{-1}^{*}\right|$. В случае, когда $E=$ $\{w:|w|=1\}$, имеем противоположное неравенство: $\left|a_{-1}\right|=0<1 / 2=\left|a_{-1}^{*}\right|$. В настоящей работе доказывается

Теорема 1. Для определенных выше функиий $f$ u $f^{*}$ справедливо неравенство

$$
\left|a_{1}\right|^{2}-\operatorname{Re} a_{1} a_{-1} \geqslant\left|a_{1}^{*}\right|^{2}-\operatorname{Re} a_{1}^{*} a_{-1}^{*} .
$$

Неравенство (2) имеет следующую емкостную интерпретацию. Пусть множество $E$ симметрично относительно вещественной оси, и пусть $H \backslash E$ - односвязная область, $H:=\{w: \operatorname{Im} w>0\}$. Обозначим через $g$ функцию, которая конформно и однолистно отображает область $H \backslash E$ на полуплоскость $H$ так, что

$$
\lim _{w \rightarrow \infty}[g(w)-w]=0
$$

Предел

$$
\operatorname{hcap}(E \cap H)=\lim _{w \rightarrow \infty} w[g(w)-w]
$$

называется относительной емкостъю множества $E \cap H$ (half-plane capacity) [2, c. 69]. Учитывая разложение функции $f$, заключаем, что функция $g$ в окрестности бесконечности имеет вид

$$
g(w)=w+\frac{a_{1}^{2}-a_{1} a_{-1}}{w}+\cdots,
$$

где $a_{1}$ и $a_{-1}-$ вещественные числа. Таким образом,

$$
\operatorname{hcap}(E \cap H)=\left|a_{1}\right|^{2}-\operatorname{Re} a_{1} a_{-1},
$$

и неравенство (2) запишется в виде

$$
\operatorname{hcap}(E \cap H) \geqslant \operatorname{hcap}\left(E^{*} \cap H\right) .
$$

Теорему 1 дополняет следующее утверждение.

ТЕОРЕма 2. Пусть функиия $\widetilde{f}(z)=\widetilde{a}_{1} z+\widetilde{a}_{0}+\widetilde{a}_{-1} / z+\cdots$ конформно и однолистно отображает область $D$ на внешность континуума $\widetilde{E} \subset E^{*}$. Тогда

$$
\left|a_{1}^{*}\right|^{2}-\operatorname{Re} a_{1}^{*} a_{-1}^{*} \geqslant\left|\widetilde{a}_{1}\right|^{2}-\operatorname{Re} \widetilde{a}_{1} \widetilde{a}_{-1} .
$$


Доказательства теорем 1, 2 будут даны в заключительной части статьи. При этом для получения неравенства (2) нам потребуется симметризация относительно окружности [3], представленная в § 2. Неравенство (3) вытекает из результата Шиффера, установленного с помощью формулы Адамара для вариации функции Грина $[4, \S 3]$. В качестве приложений теорем 1,2 приведем утверждения о покрытии в известном классе $\Sigma$ функций $f(z)=z+a_{0}+a_{-1} / z+\cdots$, мероморфных и однолистных в области $D[5]$.

СлеДСтвиЕ 1. Пусть функция $f$ принадлежит классу $\Sigma$, и пусть $w_{0}-$ произвольная точка дополнения $E=\mathbb{C}_{w} \backslash f(D)$. Тогда для любого вещественного числа ч справедливо неравенство

$$
\frac{m_{f}^{4}\left(w_{0}, \varphi\right)+16 R_{f}^{4}\left(w_{0}\right)}{8 m_{f}^{2}\left(w_{0}, \varphi\right)} \leqslant 1+\operatorname{Re} e^{-2 i \varphi} a_{-1},
$$

где $R_{f}\left(w_{0}\right) \geqslant 0$ - радиус наибольшего круга с центром в точке $w_{0}$, принадлежащего множеству $E, a m_{f}\left(w_{0}, \varphi\right)$ - линейная мера Лебега пересечения $E$ с прямой $\left\{w=w_{0}+t e^{i \varphi}: t \in \mathbb{R}\right\}$. Знак равенства достигается для функиий $f(z)=w_{0}+e^{i \varphi} \lambda^{-1} h^{-1}\left(\lambda h\left(e^{-i \varphi} z\right)\right)$ при $h(\zeta)=\zeta+1 / \zeta$ и любом $\lambda>1$.

В частности, имеют место неравенства

$$
\frac{1}{8} m_{f}^{2}\left(w_{0}, \frac{\arg a_{-1}}{2}\right)-1 \leqslant\left|a_{-1}\right| \leqslant 1-\frac{1}{8} m_{f}^{2}\left(w_{0}, \frac{\arg a_{-1}+\pi}{2}\right) .
$$

Правое неравенство уточняет хорошо известное следствие теоремы площадей: $\left|a_{-1}\right| \leqslant 1$ [5, гл. II, §4]. Оба неравенства дополняют классическую оценку

$$
m_{f}\left(w_{0}, \varphi\right) \leqslant 4 \quad \forall \varphi
$$

вытекающую из (1). Именно,

$$
\begin{aligned}
& m_{f}\left(w_{0}, \frac{\arg a_{-1}}{2}\right) \leqslant \sqrt{8\left(1+\left|a_{-1}\right|\right)} \leqslant 4 \\
& m_{f}\left(w_{0}, \frac{\arg a_{-1}+\pi}{2}\right) \leqslant \sqrt{8\left(1-\left|a_{-1}\right|\right)} .
\end{aligned}
$$

Равенства выполняются в случае $\left|a_{-1}\right|=1, f(z)=z+w_{0}+e^{2 i \varphi} / z$. Интересно было бы получить точные оценки в случае фиксированного $\left|a_{-1}\right|$, отличного от единицы.

СлЕДСТВИЕ 2. Предположим, что при некоторых $\alpha, \beta$ и $\gamma$ для функции $f(z)=z+a_{0}+a_{-1} / z+\cdots$ класса $\Sigma$ выполняется неравенство

$$
\mu\left(\left(\mathbb{C}_{w} \backslash f(D)\right) \cap l(u)\right) \geqslant \alpha \quad \forall u, \quad \beta \leqslant u \leqslant \gamma .
$$

Тогда

$$
\operatorname{Re} a_{-1} \leqslant 1-\frac{c^{2}}{2}\left(1-k^{2}\right)
$$

где вещественные постоянные с и $k$ находятся из условия

$$
c \int_{0}^{1} \sqrt{\frac{\zeta^{2}-k^{2}}{\zeta^{2}-1}} d \zeta=\frac{\gamma-\beta}{2}-\frac{i \alpha}{2}, \quad c>0, \quad 0<k<1 .
$$


Знак равенства имеет место для функиии $f$ класса $\Sigma$, конформно и однолистно отображающей область $D$ на внешность прямоугольника со сторонами, лежащими на прямых $u=\beta, u=\gamma, \gamma-\beta<4, u$ с подходящей высотой $\alpha$.

Следствия 1, 2 получаются последовательным применением неравенств (2) и (3). Список таких утверждений легко продолжить аналогично известным приложениям симметризации Штейнера в теории функций [3], [6].

\section{§ 2. Симметризация относительно окружности}

Следуя [3, §3], обозначим через $r(w)$ регулярную ветвь функции $\zeta=i \log w$, отображающую плоскость $\mathbb{C}_{w}$ с разрезом по вещественной отрицательной полуоси на полосу $-\pi<\operatorname{Re} \zeta<\pi$. Доопределим функцию $r(w)$ на разрезе в смысле граничного соответствия. Пусть $E$ - произвольное замкнутое множество в плоскости $\overline{\mathbb{C}}_{w}$, не содержащее начало координат и бесконечно удаленную точку. Симметризацией множества $E$ относительно окружности $|w|=1$ называется переход от множества $E$ к симметричному относительно $|w|=1$ множеству

$$
R E=r^{-1}\left((r(E))^{*}\right),
$$

где, как и выше, * означает симметризацию Штейнера относительно вещественной оси, выполненную в полосе $-\pi \leqslant \operatorname{Re} \zeta \leqslant \pi$. Дадим теперь прямое определение этого преобразования. Для замкнутого множества $E$, принадлежащего $\mathbb{C}_{w} \backslash\{0\}$, введем обозначения

$$
\begin{aligned}
& E(\theta)=E \cap\{w: \arg w=\theta\}, \quad R(\theta)=\exp \left(\frac{1}{2} \int_{E(\theta)} \frac{d \rho}{\rho}\right), \\
& \widetilde{E}(\theta)= \begin{cases}\left\{w=\rho e^{i \theta}: R^{-1}(\theta) \leqslant \rho \leqslant R(\theta)\right\}, & \text { если } E(\theta) \neq \varnothing, \\
\varnothing, & \text { если } E(\theta)=\varnothing .\end{cases}
\end{aligned}
$$

Легко видеть, что

$$
R E=\bigcup_{0 \leqslant \theta \leqslant 2 \pi} \widetilde{E}(\theta) .
$$

При фиксированном $v>0$ пусть $L_{v}(w):=w / v-i-$ линейное преобразование, переводящее окружность $|w-i v|=v$ в единичную окружность $|w|=1$, и пусть $L_{v}^{-1}$ - обратное отображение. Результатом симметризации замкнутого ограниченного множества $E$ относительно окружности $|w-i v|=v$ назовем множество

$$
R_{v} E=L_{v}^{-1}\left(R L_{v}(E)\right)
$$

Для открытого множества $B$, содержащего точки $i v$ и $\infty$, положим

$$
S_{v} B=\overline{\mathbb{C}}_{w} \backslash R_{v}\left(\overline{\mathbb{C}}_{w} \backslash B\right)
$$

Лемма 1. Если открытые множества $B_{1}$ и $B_{2}$ удовлетворяют условиям $\infty \in B_{1}$ и $\bar{B}_{1} \subset B_{2}$, то для достаточно больиих $v>0$ выполняется включение

$$
S_{v} B_{1} \subset \overline{\mathbb{C}}_{w} \backslash\left(\overline{\mathbb{C}}_{w} \backslash B_{2}\right)^{*} \quad\left(R_{v}\left(\overline{\mathbb{C}}_{w} \backslash B_{1}\right) \supset\left(\overline{\mathbb{C}}_{w} \backslash B_{2}\right)^{*}\right) .
$$


Доказательство леммы 1 очевидно, носит технический характер и поэтому здесь не проводится. Отметим лишь важность того факта, что множество $B_{1}$ лежит компактно в $B_{2}$. В этом случае замкнутое множество $\overline{\mathbb{C}}_{w} \backslash B_{2}$ принадлежит $\overline{\mathbb{C}}_{w} \backslash B_{1}$ вместе с некоторой окрестностью $U$. Лучи, проходящие через точку $i v$ и пересекающие окрестность $U$, стремятся при $v \rightarrow \infty$ вблизи вещественной оси к прямым, параллельным мнимой оси. При этом “логарифмическая мера" в окрестности окружности $|w-i v|=v$ стремится к евклидовой.

Обозначим через $g_{B}\left(z, z_{0}\right)$ функцию Грина связной компоненты открытого множества $B$, содержащей точку $z_{0}$ (с полюсом в этой точке), доопределенную нулем вне этой связной компоненты. Пусть $r\left(B, z_{0}\right)$ - внутренний радиус указанной компоненты относительно точки $z_{0}[3]$.

ЛЕмма 2. Если связные компоненты открытого множества В имеют функиии Грина, а точки iv и $\infty$ принадлежат $B$, то

$$
\log [r(B, i v) r(B, \infty)]+2 g_{B}(i v, \infty) \leqslant \log \left[r\left(S_{v} B, i v\right) r\left(S_{v} B, \infty\right)\right]+2 g_{S_{v} B}(i v, \infty)
$$

для любого $v>0$.

ДокАЗАТЕЛЬство вытекает из [3, теорема 1.7, предложение 1.11] (см. также [7, теорема 1]).

\section{§ 3. Доказательства}

ДОКАЗАТЕЛЬСТВо ТЕОРЕмЫ 1 . Пусть $\left\{B_{n}\right\}_{n=1}^{\infty}-$ исчерпание области $f(D)$ односвязными областями $B_{n}, \infty \in B_{n}, \bar{B}_{n} \subset B_{n+1}, n=1,2, \ldots, \bigcup_{n=1}^{\infty} B_{n}=$ $f(D)$. Для каждого $n$ и достаточно большого $v>0$ функция $u_{n}(w):=$ $g_{B_{n}}(w, i v)-g_{B_{n}}(w, \infty)$ является гармонической в области $B_{n} \backslash\{i v, \infty\}$, причем $u_{n}(w) \rightarrow+\infty$ при $w \rightarrow i v$ и $u_{n}(w) \rightarrow-\infty$, когда $w \rightarrow \infty$. Отсюда следует, в частности, что множества $B_{n}^{1}:=\left\{w: u_{n}(w)>0\right\}$ и $B_{n}^{2}:=\left\{w: u_{n}(w)<0\right\}$ являются непересекающимися областями. Функция Грина области $B_{n}^{1}$ с полюсом в точке $i v$ совпадает в $B_{n}^{1}$ с функцией $u_{n}(w)$. Следовательно,

$$
\log r\left(B_{n}^{1}, i v\right)=\lim _{w \rightarrow i v}\left(u_{n}(w)+\log |w-i v|\right)=\log r\left(B_{n}, i v\right)-g_{B_{n}}(i v, \infty) .
$$

Аналогично, функция $-u_{n}(w)$ совпадает в $B_{n}^{2}$ с функцией Грина этой области с полюсом в точке $w=\infty$. Поэтому

$$
\log r\left(B_{n}^{2}, \infty\right)=\lim _{w \rightarrow \infty}\left(-u_{n}(w)-\log |w|\right)=\log r\left(B_{n}, \infty\right)-g_{B_{n}}(\infty, i v) .
$$

Суммируя полученные соотношения, имеем

$$
\begin{gathered}
\log \left[r\left(B_{n}, i v\right) r\left(B_{n}, \infty\right)\right]-2 g_{B_{n}}(i v, \infty)=\log \left[r\left(B_{n}^{1}, i v\right) r\left(B_{n}^{2}, \infty\right)\right] \\
=\log \left[r\left(B_{n}^{1} \cup B_{n}^{2}, i v\right) r\left(B_{n}^{1} \cup B_{n}^{2}, \infty\right)\right]+2 g_{B_{n}^{1} \cup B_{n}^{2}}(i v, \infty) .
\end{gathered}
$$

По лемме 2 последнее выражение не превосходит суммы

$$
\log \left[r\left(S_{v}\left(B_{n}^{1} \cup B_{n}^{2}\right), i v\right) r\left(S_{v}\left(B_{n}^{1} \cup B_{n}^{2}\right), \infty\right)\right]+2 g_{S_{v}\left(B_{n}^{1} \cup B_{n}^{2}\right)}(i v, \infty) .
$$


Поскольку $B_{n}^{1} \cap B_{n}^{2}=\varnothing$, для любого $\theta$ луч $w=i v+\rho e^{i \theta}, 0 \leqslant \rho \leqslant \infty$, пересекает множество $\overline{\mathbb{C}}_{w} \backslash\left(B_{n}^{1} \cup B_{n}^{2}\right)$. Следовательно, по определению симметризации относительно окружности множество $R_{v}\left(\overline{\mathbb{C}}_{w} \backslash\left(B_{n}^{1} \cup B_{n}^{2}\right)\right)$ содержит окружность $|w-i v|=v$. Поэтому связные компоненты $\widetilde{B}_{n}^{1}$ и $\widetilde{B}_{n}^{2}$ множества $S_{v}\left(B_{n}^{1} \cup B_{n}^{2}\right)$, содержащие соответственно точки $i v$ и $w=\infty$, не пересекаются, и мы имеем

$$
\begin{gathered}
r\left(S_{v}\left(B_{n}^{1} \cup B_{n}^{2}\right), i v\right)=r\left(\widetilde{B}_{n}^{1}, i v\right), \quad r\left(S_{v}\left(B_{n}^{1} \cup B_{n}^{2}\right), \infty\right)=r\left(\widetilde{B}_{n}^{2}, \infty\right), \\
g_{S_{v}\left(B_{n}^{1} \cup B_{n}^{2}\right)}(i v, \infty)=g_{\widetilde{B}_{n}^{1} \cup \widetilde{B}_{n}^{2}}(i v, \infty)=0 .
\end{gathered}
$$

Окончательно получаем

$$
\begin{aligned}
\log \left[r\left(B_{n}, i v\right) r\left(B_{n}, \infty\right)\right] & -2 g_{B_{n}}(i v, \infty) \leqslant \log \left[r\left(\widetilde{B}_{n}^{1}, i v\right) r\left(\widetilde{B}_{n}^{2}, \infty\right)\right]-2 g_{\widetilde{B}_{n}^{1} \cup \widetilde{B}_{n}^{2}}(i v, \infty) \\
= & \log \left[r\left(S_{v} B_{n}, i v\right) r\left(S_{v} B_{n}, \infty\right)\right]-2 g_{S_{v} B_{n}}(i v, \infty) .
\end{aligned}
$$

Последнее равенство устанавливается аналогично (4) с учетом симметрии области $S_{v} B_{n}$ относительно окружности $|w-i v|=v$. Далее воспользуемся следующим фактом, доказанным впервые Шиффером $[4, \S 3]:$ если области $G_{1}$ и $G_{2}$ имеют функции Грина, $G_{1} \subset G_{2}$ и $\zeta, w-$ различные точки области $G_{1}$, то справедливо неравенство

$$
\log \left[r\left(G_{1}, \zeta\right) r\left(G_{1}, w\right)\right]-2 g_{G_{1}}(\zeta, w) \leqslant \log \left[r\left(G_{2}, \zeta\right) r\left(G_{2}, w\right)\right]-2 g_{G_{2}}(\zeta, w) .
$$

По лемме 1 имеем

$$
S_{v} B_{n} \subset \overline{\mathbb{C}}_{w} \backslash\left(\overline{\mathbb{C}}_{w} \backslash f(D)\right)^{*} \subset f^{*}(D) .
$$

Таким образом, из (5) и (6) следует неравенство

$$
\begin{aligned}
\log [ & \left.r\left(B_{n}, i v\right) r\left(B_{n}, \infty\right)\right]-2 g_{B_{n}}(i v, \infty) \\
& \leqslant \log \left[r\left(f^{*}(D), i v\right) r\left(f^{*}(D), \infty\right)\right]-2 g_{f^{*}(D)}(i v, \infty) .
\end{aligned}
$$

Обозначим через

$$
f_{n}(z)=a_{1}^{n} z+a_{0}^{n}+\frac{a_{-1}^{n}}{z}+\cdots
$$

функцию, конформно и однолистно отображающую область $D$ на область $B_{n}$, и пусть

$$
h_{n}(w)=\frac{w}{a_{1}^{n}}-\frac{a_{0}^{n}}{a_{1}^{n}}-\frac{a_{-1}^{n}}{w}+\cdots
$$

- разложение обратного отображения в окрестности бесконечно удаленной точки. Тогда при достаточно большом $v$ выполняются равенства

$$
\begin{gathered}
r\left(B_{n}, \infty\right)\left|a_{1}^{n}\right|=r(D, \infty)=1, \quad r\left(B_{n}, i v\right)\left|h_{n}^{\prime}(i v)\right|=r\left(D, h_{n}(i v)\right)=\left|h_{n}(i v)\right|^{2}-1, \\
g_{B_{n}}(i v, \infty)=g_{D}\left(h_{n}(i v), \infty\right)=\log \left|h_{n}(i v)\right| .
\end{gathered}
$$

Отсюда имеем

$$
\begin{aligned}
& r\left(B_{n}, i v\right) r\left(B_{n}, \infty\right) e^{-2 g_{B_{n}}(i v, \infty)}=\frac{\left|h_{n}(i v)\right|^{2}-1}{\left|a_{1}^{n} h_{n}^{\prime}(i v)\right|\left|h_{n}(i v)\right|^{2}} \\
& \quad=\frac{1-\left|\frac{i v}{a_{1}^{n}}+O(1)\right|^{-2}}{\left|1-\frac{a_{1}^{n} a_{-1}^{n}}{v^{2}}+o\left(\frac{1}{v^{2}}\right)\right|}=1-\left(\left|a_{1}^{n}\right|^{2}-\operatorname{Re} a_{1}^{n} a_{-1}^{n}\right) \frac{1}{v^{2}}+o\left(\frac{1}{v^{2}}\right), \quad v \rightarrow+\infty .
\end{aligned}
$$


Повторяя предыдущие выкладки для функции $f^{*}$ вместо $f_{n}$, из неравенства $(7)$ получаем

$$
\left|a_{1}^{n}\right|^{2}-\operatorname{Re} a_{1}^{n} a_{-1}^{n} \geqslant\left|a_{1}^{*}\right|^{2}-\operatorname{Re} a_{1}^{*} a_{-1}^{*} .
$$

Переходя к пределу при $n \rightarrow \infty$, получаем неравенство (2). Теорема доказана.

ДокАЗАТЕЛЬСтво тЕОРЕмЫ 2 вытекает из неравенства (6), где необходимо положить $G_{1}=f^{*}(D), G_{2}=\widetilde{f}(D), \zeta=i v, w=\infty$ и где $v$ достаточно большое. Далее приводятся вычисления для функций $f^{*}$ и $\widetilde{f}$, повторяющие концовку доказательства теоремы 1 для функции $f_{n}$. В итоге получаем неравенство (3).

ДокАЗАТЕЛЬСТВо СЛЕДСТВИЯ 1 . Рассмотрим сначала случай, когда $w_{0}=0$ и $\varphi=\pi / 2$. Функция

$$
\tilde{f}(z):=i R_{f}(0) h^{-1}(\lambda h(z))=i \lambda R_{f}(0) z+\frac{i R_{f}(0)}{z}\left(\lambda-\frac{1}{\lambda}\right)+\cdots
$$

при $\lambda=\left[m_{f}^{2}(0, \pi / 2) /\left(4 R_{f}^{2}(0)\right)+1\right]\left[m_{f}(0, \pi / 2) / R_{f}(0)\right]^{-1}$ конформно и однолистно отображает область $D$ на дополнение к множеству $\widetilde{E}:=\left\{w:|w| \leqslant R_{f}(0)\right\} \cup$ $\left\{w: \operatorname{Re} w=0,|\operatorname{Im} w| \leqslant m_{f}(0, \pi / 2) / 2\right\}$. В условиях следствия имеем $\widetilde{E} \subset E^{*}$ $\left(E=\overline{\mathbb{C}}_{w} \backslash f(D)\right)$. Поэтому из неравенств $(2)$ и (3) получаем

$$
1-\operatorname{Re} a_{-1} \geqslant R_{f}^{2}(0)\left(2 \lambda^{2}-1\right)=\frac{m_{f}^{4}(0, \pi / 2)+16 R_{f}^{4}(0)}{8 m_{f}^{2}(0, \pi / 2)} .
$$

В случае произвольных $w_{0}$ и $\varphi$ необходимо применить предыдущее заключение к функции

$$
e^{i(\pi / 2-\varphi)}\left[f\left(e^{i(\varphi-\pi / 2)} z\right)-w_{0}\right]=z+\tilde{a}_{0}-e^{-2 i \varphi} \frac{a_{-1}}{z}+\cdots .
$$

Условие равенства проверяется непосредственно.

ДокАЗАТЕЛЬСТво СЛЕДСТВИЯ 2. Можно считать, что $\beta=-\gamma$. Функция

$$
F(\zeta)=c \int_{0}^{\zeta} \sqrt{\frac{\zeta^{2}-k^{2}}{\zeta^{2}-1}} d \zeta+\frac{i \alpha}{2}=c \zeta+c_{0}-\frac{c\left(1-k^{2}\right)}{2 \zeta}+\cdots
$$

конформно и однолистно отображает верхнюю полуплоскость $\operatorname{Im} \zeta>0$ на четырехугольник $\{w: \operatorname{Im} w>0\} \backslash \widetilde{E}$, где на этот раз $\widetilde{E}=\{w:|\operatorname{Re} w| \leqslant \gamma,|\operatorname{Im} w| \leqslant$ $\alpha / 2\}$. По принципу симметрии Римана-Шварца функция

$$
\widetilde{f}(z):=F\left(\frac{1}{2}\left(z+\frac{1}{z}\right)\right)=\frac{c}{2} z+c_{0}-c\left(\frac{1}{2}-k^{2}\right) \frac{1}{z}+\cdots
$$

конформно и однолистно отображает область $D$ на внешность прямоугольника $\widetilde{E}$. В условиях следствия имеем $\widetilde{E} \subset\left(\overline{\mathbb{C}}_{w} \backslash f(D)\right)^{*}$. Осталось воспользоваться неравенствами (2) и (3).

\section{Список литературы}

1. Г. Полиа, Г. Сегё, Изопериметрические неравенства в математической физике, ГИФМЛ, М., 1962; пер. с англ.: G. Pólya, G. Szegő, Isoperimetric inequalities in mathematical physics, Ann. of Math. Stud., 27, Princeton Univ. Press, Princeton, NJ, 1951. 
2. G.F. Lawler, Conformally invariant processes in the plane, Math. Surveys Monogr., 114, Amer. Math. Soc., Providence, RI, 2005.

3. В.Н. Дубинин, "Симметризация в геометрической теории функций комплексного переменного", УМH, 49:1 (1994), 3-76; англ. пер.: V. N. Dubinin, "Symmetrization in the geometric theory of functions of a complex variable", Russian Math. Surveys, 49:1 (1994), 1-79.

4. М. Шиффер, Некоторые новые результаты в теории конформных отображений, Приложение к кн.: Курант Р., Принцип Дирихле, конформные отображения и минимальные поверхности, ИЛ, М., 1953; пер. с англ.: М. Schiffer, Appendix: R. Courant, Dirichlet's Principle, conformal mapping, and minimal surfaces, Interscience Publ., New York, 1950.

5. Г. М. Голузин, Геометрическая теория функиий комплексного переменного, Наука, M., 1966; англ. пер.: G. M. Goluzin, Geometric theory of functions of a complex variable, Transl. Math. Monogr., 26, Amer. Math. Soc., Providence, RI, 1969.

6. В. К. Хейман, Многолистные функиии, ИЛ, М., 1960; пер. с англ.: W. К. Hayman, Multivalent functions, Cambridge Univ. Press, Cambridge, 1958.

7. В.Н.Дубинин, "Некоторые свойства внутреннего приведенного модуля", Сиб. матем. журн., 35:4 (1994), 774-792; англ. пер.: V. N. Dubinin, "Some properties of the reduced inner modulus", Siberian Math. J., 35:4 (1994), 689-705.

B. Н. Дувинин (V. N. Dubinin)

Институт прикладной математики ДВО РАН, г. Владивосток

E-mail: dubinin@iam.dvo.ru
Поступило в редакцию 27.01.2009 